\title{
A two-warehouse production inventory model with trade credit under reliability consideration
}

\author{
Pinky Saxena ${ }^{a^{*}}$, S. R. Singh ${ }^{b}$ and Isha Sangal ${ }^{c}$
}

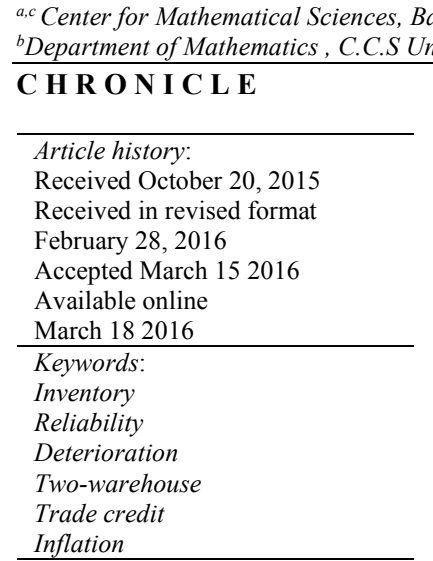

\section{Introduction}

In classical economic production quantity model, it is assumed that the production set-up cost is fixed and all the items produced are of perfect quality. But, in reality all the items produced are not always perfect but directly affected by the reliability of the production process. The quality of the product can be improved by investment in the reliability of the production process. During the past few years, researchers have started focusing on this concept. Lin and Hou (2005) presented a model with improvement in process reliability, quality and reduction in set up time. Cheng (1989) developed economic production quantity model with flexibility and reliability consideration. Pal et al. (2007) focused on policy with variable demand under reliability consideration. Leung (2007) generalized geometric programming solution to economic production quantity model with flexibility and reliability consideration. Yadav et al. (2010) focused on production model under limited storage with flexibility and reliability consideration. Singhal (2013) developed volume flexible inventory system in Fuzzy environment. Panda and Maiti (2009) developed a multi-item inventory model with price dependent demand with flexibility and reliability consideration under fuzzy environment using geometric programming approach. In today's competitive environment, it is more common to see that retailer is

\footnotetext{
* Corresponding author

E-mail address: pinkysaxena@gmail.com (P. Saxena) 
allowed a fixed time period before they settle their account to the supplier. This period is also known as trade credit period. Before the end of the end of trade credit period the retailer can sell the goods and accumulate revenue and earn interest. A high interest is charged if the payment is not settled by the end of this period. Shah (2006) developed an inventory model for items deteriorating with time under permissible delay in period. Soni et al. (2006) presented an EOQ model under DCF approach for progressive payment scheme. Yang (2006) developed a two warehouse inventory model under conditionally delay in payment. He focused on warehouse problem considering partial backlogging. Singh and Saxena (2014) presented two-warehouse inventory model under trade credit in fuzzy environment.

The effect of inflation is observed in many countries; first, it has been introduced by Buzacot (1975) where economic order quantity model under inflation is presented. Several researchers extended the work of in different ways. Dey et al. (2008) developed two storage inventory models under inflation and time value of money. Singh et al. (2009) highlighted an integrated model with multivariable demand with credit period. Hadidi et al. (2011) focused on integrated cost model under perfect maintenance. Soni et al. (2006) illustrated optimal policies involving various service level constraints. Park (1983) presented integrated production model for deteriorating item. Yan and Cheng (1988) developed economic order quantity model under assumption of production stopping and restarting times. Maity and Maiti (2009) presented optimal inventory policies under different substitute item with deterioration. Singh et al. (2013) illustrated an EOQ model considering trapezoidal demand and trade credit. They presented economic order quantity model with variable demand rate under inflation. Tayal et al. (2014) developed a production inventory model under space restriction. Singh and Prasher (2014) presented production inventory model considering machine breakdown and stochastic repair time.

In this paper we have presented two warehouse production inventory models for deteriorating items with permissible delay in payment under inflation and reliability consideration. Demand rate is considered as an exponential function of time. Finally, some numerical examples for illustration are provided and sensitivity analysis is performed.

\section{Assumptions and Notations}

\subsection{Assumptions}

- Production rate is greater than demand rate. Also, it is linear combination of on-hand inventory and demand rate i.e. $P(t)=[I(t)+b D(t)]\left(1-e^{-d t}\right)$.

- Demand rate is exponentially an increasing function of time i.e. $D(t)=\mu e^{\lambda t}, 0 \leq \lambda \leq 1$.

- Deterioration is taken as time dependent for owned warehouse, while, Wei-bull distribution for rented warehouse.

- Planning Horizon is finite.

- Model is considered for imperfect items and inflation is also taken in this model.

- Shortages are not allowed.

- Lead time is zero, and no replenishment or repair of deteriorated items is made during a given cycle.

- A single item is considered over the prescribed period $\mathrm{T}$ units of time, which is subject to variable deterioration rate.

- The owned warehouse (O.W) has a fixed capacity of W units, and the rented warehouse (R.W) has unlimited capacity.

- The supplier provides the retailer a permissible delay of payments. During the trade credit period the account is not settled, the revenue is deposited in an interest bearing account. At the end of the permissible delay, the retailer pays off the items ordered, and starts to pay the interest charged on the items in stock. 
- Total cost of interest and depreciation per production cycle is inversely related to the set up cost and directly related to process reliability i.e. $\mathrm{f}\left(\mathrm{C}_{\mathrm{s}}, \mathrm{v}\right)=\mathrm{c} \mathrm{C}_{\mathrm{s}}{ }^{-\mathrm{e}} \mathrm{v}^{\mathrm{f}}$ where $\mathrm{c}, \mathrm{e}, \mathrm{f}>0$ are constant real numbers chosen to provide the best fit of the estimated cost function. The process reliability level $\mathrm{v}$ means of all the items produced in a production run only $\mathrm{v} \%$ are acceptable quality that can be used to meet demand.

\subsection{Notations}

$\begin{array}{ll}d & \text { Imperfect production rate } \\ W & \text { Owned warehouse capacity } \\ C_{S} & \text { Set up cost per production run. (a decision variable) } \\ C_{R W} & \text { Holding cost rented warehouse per unit time } \\ C_{O W} & \text { Holding cost owned warehouse per unit time } \\ C_{D} & \text { Deterioration cost } \\ I_{O_{1}}(t) & \text { Inventory level in owned warehouse } 0 \leq \mathrm{t} \leq \mathrm{t}_{1} \\ I_{R_{2}}(t) & \text { Inventory level in rented warehouse } \mathrm{t}_{1} \leq \mathrm{t} \leq \mathrm{t}_{2} \\ I_{R_{3}}(t) & \text { Inventory level in rented warehouse } \mathrm{t}_{2} \leq \mathrm{t} \leq \mathrm{t}_{3} \\ I_{O_{4}}(t) & \text { Inventory level in owned warehouse } \mathrm{t}_{3} \leq \mathrm{t} \leq \mathrm{T} \\ I_{O_{5}}(t) & \text { Inventory level in owned warehouse } \mathrm{t}_{1} \leq \mathrm{t} \leq \mathrm{t}_{3} \\ t_{1}, t_{2} & \text { Production period for owned warehouse and rented warehouse } \\ t_{3}, T & \text { Non-Production period } \\ T & \text { Total cycle time } \\ M & \text { Retailer's trade credit period offered by supplier in years } \\ S & \text { Unit selling price } \\ C & \text { Unit purchase cost } \\ I_{e} & \text { Interest which can be earned per } \$ \text { per year } \\ I_{C} & \text { Interest charges per } \$ \text { in stocks per year by the supplier } \\ I D C & \text { Cost of interest and depreciation per production cycle } \\ T C_{1}(C S, v, T) & \text { Present worth of total cost per unit time, when } \mathrm{M} \leq \mathrm{t}_{3}<\mathrm{T} \\ T C_{2}(C S, v, T) & \text { Present worth of total cost per unit time, when } \mathrm{t}_{3}<\mathrm{M} \leq \mathrm{T} \\ T C_{3}(C S, v, T) & \text { Present worth of total cost per unit time, when } \mathrm{M}>\mathrm{T}\end{array}$

\section{Mathematical model}

The production starts at time $t=0$, and items accumulate from 0 up to $W$ units in owned warehouse. After time $t_{1}$ any production quantity exceeding $\mathrm{W}$ will be stored in rented warehouse. After this production stopped and the inventory level in rented warehouse begins to decrease at $t_{2}$ and will reach 0 units at $t_{3}$ due to combined effect of demand and deterioration. The inventory level in owned warehouse comes to decrease at $t_{1}$ and then falls below $\mathrm{W}$ at $t_{2} \& t_{3}$ due to deterioration. But, during $\left[\mathrm{t}_{3}, \mathrm{~T}\right]$, the inventory is depleted due to both demand and deterioration. By the time to $\mathrm{T}$, both warehouses are empty Fig. 1 shows the behavior of inventory system.

These are the differential equations showing the inventory with the variation in time.

$$
\begin{array}{ll}
\frac{d I_{O_{1}}(t)}{d t}+\theta I_{O_{1}}(t)=P(t)-D(t) & 0 \leq t \leq t_{1} \\
\frac{d I_{R_{2}}(t)}{d t}+\theta I_{R_{2}}(t)=P(t)-D(t) & t_{1} \leq t \leq t_{2}
\end{array}
$$




$$
\begin{array}{ll}
\frac{d I_{R_{3}}(t)}{d t}+\theta I_{R_{3}}(t)=-D(t) & t_{2} \leq t \leq t_{3} \\
\frac{d I_{O_{4}}(t)}{d t}+\theta I_{O_{4}}(t)=-D(t) & t_{3} \leq t \leq T \\
\frac{d I_{O_{5}}(t)}{d t}+\theta I_{O_{5}}(t)=0 & t_{1} \leq t \leq t_{3}
\end{array}
$$

With boundary conditions:

$$
I_{Q}(0)=0, I_{R_{2}}\left(t_{1}\right)=0, I_{R_{3}}\left(t_{3}\right)=0, I_{O_{4}}(T)=0, I_{O_{3}}\left(t_{1}\right)=W
$$

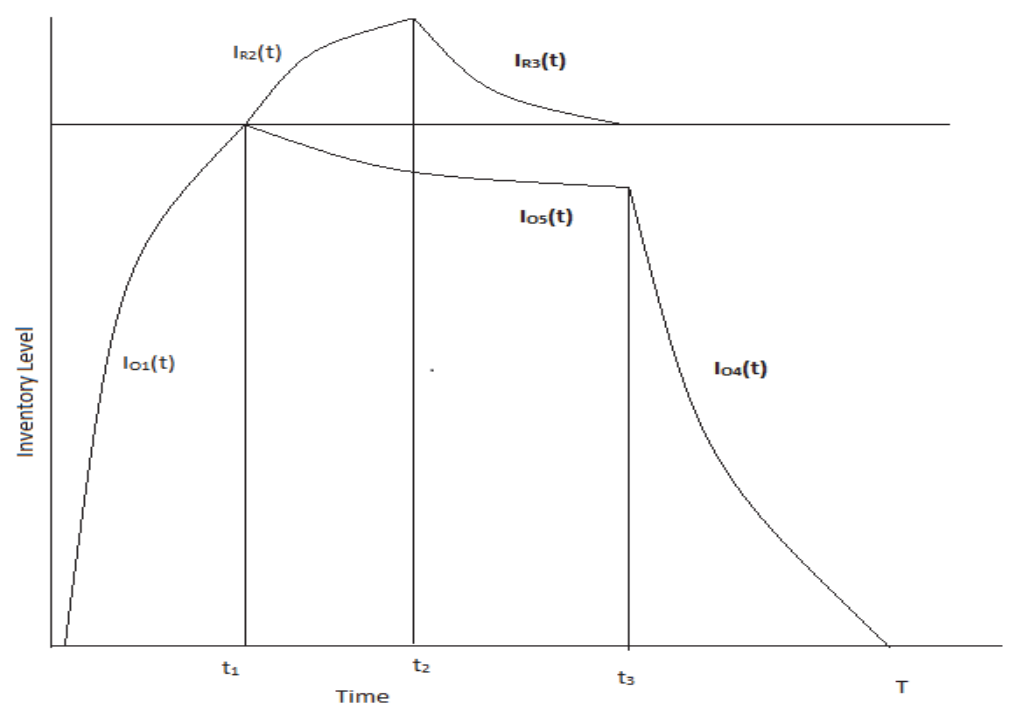

Fig. 1. Graphical representation of the two-warehouse inventory system

The solution of these above mentioned equations are given as follow:

$$
\begin{array}{ll}
I_{Q}(t)=(b-1) \mu\left(\frac{d t^{2}}{2}+\frac{\lambda d t^{3}}{3}-\frac{(\theta-d) d t^{4}}{4}\right)-\mu\left(t+\frac{\lambda t^{2}}{2}-(\theta-d) \frac{t^{3}}{2}\right) & 0 \leq t \leq t_{1} \\
I_{R_{2}}(t)=(b-1) \mu\left(\frac{d\left(t^{2}-t_{1}^{2}\right)}{2} \frac{\alpha d t^{\beta}\left(t^{2}-t_{1}^{2}\right)}{2}+\frac{\left(t^{3}-t_{1}^{3}\right) \lambda d}{3}\right)-\mu\left(\left(t-t_{1}\right)-\alpha t^{\beta}\left(t-t_{1}\right)+\frac{\lambda\left(t^{2}-t_{1}^{2}\right)}{2}-\left(t^{2}-t_{1}^{2}\right) \frac{\lambda \alpha t^{\beta}}{2}\right), & t_{1} \leq t \leq t_{2} \\
I_{R_{3}}(t)=\mu\left(\left(t_{3}-t\right)-\alpha t^{\beta}\left(t_{3}-t\right)+\frac{\lambda}{2}\left(t_{3}^{2}-t^{2}\right)-\frac{\lambda \alpha t^{\beta}}{2}\left(t_{3}^{2}-t^{2}\right)\right) & t_{2} \leq t \leq t_{3} \\
I_{O_{t}}(t)=\mu\left((T-t)-\frac{\theta t^{2}}{2}(T-t)+\frac{\lambda}{2}\left(T^{2}-t^{2}\right)-\frac{\lambda \theta t^{2}}{4}\left(T^{2}-t^{2}\right)\right) & t_{3} \leq t \leq T \\
I_{O_{5}}(t)=W e^{-\left(\frac{\theta}{2}\right)\left(t_{1}^{2}-t^{2}\right)} & t_{1} \leq t \leq t_{3}
\end{array}
$$

Based on the assumptions and description of the model, the total annual costs, TC, include the following elements:

The present worth ordering cost is given by $\mathrm{C}_{\mathrm{S}}$

The present worth inventory holding cost in rented warehouse and owned warehouse are 
Holding Cost rented warehouse $\quad=c_{R W}\left(\int_{t_{1}}^{t_{2}} I_{R_{2}}(t) e^{-r t} d t+\int_{t_{2}}^{t_{3}} I_{R_{3}}(t) e^{-r t} d t\right)$

Holding Cost owned warehouse $\quad=c_{O W}\left(\int_{0}^{t_{1}} I_{O_{1}}(t) e^{-r t} d t+\int_{t_{1}}^{t_{3}} I_{O_{5}}(t) e^{-r t} d t+\int_{t_{3}}^{T} I_{O_{4}}(t) e^{-r t} d t\right)$

The present worth deterioration cost $=$

$$
\begin{aligned}
& I_{D}=c_{D}\left(\int_{0}^{t_{1}} \theta t I_{O_{1}}(t) e^{-r t} d t+\int_{t_{1}}^{t_{3}} \theta t I_{O_{5}}(t) e^{-r t} d t+\int_{t_{3}}^{T} \theta t I_{O_{4}}(t) e^{-r t} d t+\int_{t_{1}}^{t_{2}} \alpha \beta t^{\beta-1} I_{R_{2}}(t) e^{-r t} d t\right. \\
& \left.+\int_{t_{2}}^{t_{3}} \alpha \beta t^{\beta-1} I_{R_{3}}(t) e^{-r t} d t\right)
\end{aligned}
$$

Now,we will calculate interest paid and earned by the retailer, for this there are three cases:

Case 1: $\left(\mathrm{M} \leq \mathrm{t}_{3}<\mathrm{T}\right)$

In this case, the permissible delay period $M$ expires before the total inventory depletion period $T$. Therefore, retailer will have to pay interest charged on unsold items during $(M, T)$.

In this case, $M \leq T$ the present worth of interest earned is

$$
I E_{1}=s I_{e} \int_{0}^{M} D t \mathrm{e}^{-\mathrm{rt}} d t=\mu s I_{e}\left(\frac{M^{2}}{2}-(r-\lambda) \frac{M^{3}}{3}+\frac{\lambda r M^{4}}{4}\right)
$$

Hence, present worth of interest payable by retailer is given by

$$
I C_{1}=c I_{C}\left(\int_{M}^{t_{3}} I_{R_{3}}(t) e^{-r t} d t+\int_{M}^{t_{3}} I_{O_{5}}(t) e^{-r t} d t+\int_{t_{3}}^{T} I_{O_{4}}(t) e^{-r t} d t\right)
$$

Case 2: $\left(t_{3}<M \leq T\right)$ In this case, present worth interest payable is

$$
I C_{2}=c I_{C} \int_{M}^{T} I_{O_{4}}(t) e^{-r t} d t
$$

Case 3: $(M>T)$, In this case, no interest charges are paid for the items

$$
I C_{3}=0
$$

Hence, the present worth interest earned is given by

$$
I E_{2}=s I_{e}\left(\int_{0}^{T} D t e^{-r t} d t+D T(M-T)\right)
$$

Therefore, the present worth annual total relevant costs for the retailer can be expressed as 
$\mathrm{TC}\left(\mathrm{t}_{3}, \mathrm{C}_{\mathrm{S}}, \mathrm{v}, \mathrm{T}\right)=$ Ordering Cost + Holding cost in Rented Warehouse + Holding cost in Owned

Warehouse + Deteriorating cost + Depreciation Cost + Interest payable cost -Interest earned.

Therefore, the total cost per unit time of the given inventory model as a function of $t_{1}, t_{2}, t_{3}, v, C_{S}$ and $T$ say $T C\left(t_{1}, t_{2}, t_{3}, v, C_{S}, T\right)$ is given by

$$
\mathrm{TC}\left(\mathrm{t}_{3}, C S, \mathrm{v}, \mathrm{T}\right)=\left\{\begin{array}{l}
T C_{1}, \text { if } M \leq t_{3}<T \\
T C_{2}, \text { if } t_{3}<M \leq T \\
T C_{3}, \text { if } M>T
\end{array}\right.
$$

Eq. (20) denotes the cost function of the system in terms of $t_{1}, t_{2}, t_{3}, v, C_{S}$ and $T$. To find out the optimal solution of this system we have to find out the optimal values of $t_{1}, t_{2}, t_{3}, C_{S}, v$ and $T$. We have relation between these variables.

$$
0 \leq t_{1} \leq t_{2} \leq t_{3} \leq T
$$

\section{Numerical Analysis}

The above discussed inventory model is illustrated through the numerical example for which the input values are considered in proper units as follows:

$$
\begin{aligned}
& \theta=0.01, r=1, \lambda=2, \mu=20, C_{D}=2, I_{C}=0.15, M=0.25, c=0.2, f=1, e=1 \\
& \alpha=0.06, \beta=0.01, d=0.1, b=5, C_{R W}=0.6, W=500, C_{O W}=1.2, C=0.2
\end{aligned}
$$

There are three cases according to the permissible delay period as follows:

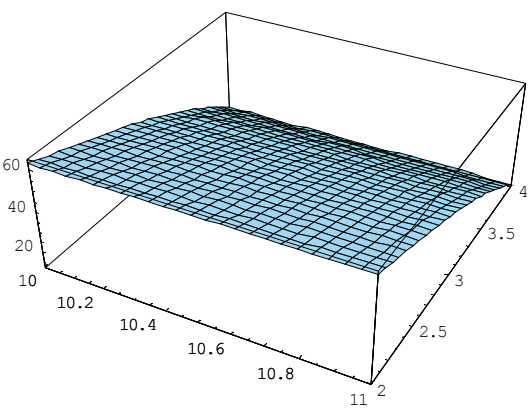

Fig.2. (Case: $\mathrm{M} \leq \mathrm{t} 3<\mathrm{T}$ ) Convexity of TC 1 w.r.t. $v$ and $\mathrm{Cs}$

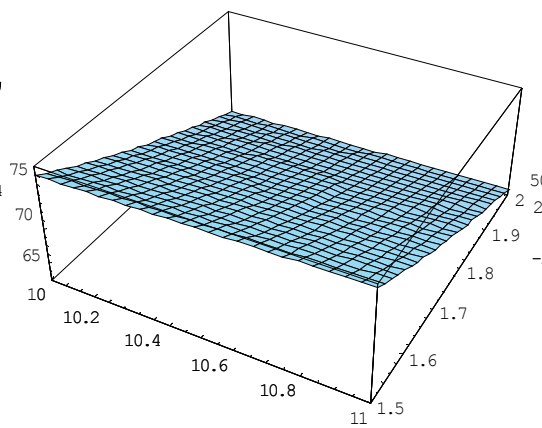

Fig. 3. (Case: $\mathrm{t} 3<\mathrm{M}<\mathrm{T}$ ) Convexity of TC 2 w.r.t. $v$ and Cs

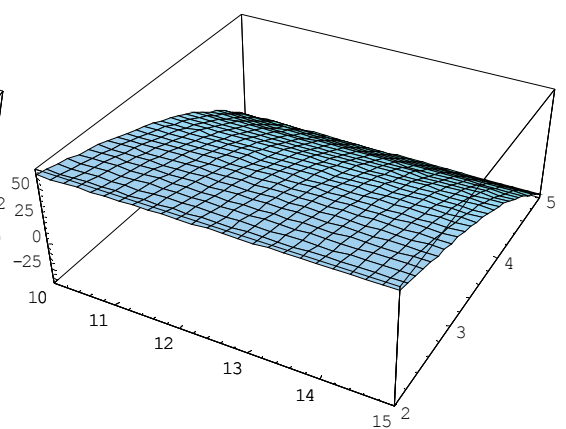

Fig.4. (Case: $\mathrm{M}>\mathrm{T}$ ) Convexity of TC 3 w.r.t. $v$ and $\mathrm{Cs}$

Case 1: $\left(\mathrm{M} \leq \mathrm{t}_{3}<\mathrm{T}\right)$, Using mathematical software Mathematica 7 the output results are as follows: $\mathrm{T}=1.56547, \mathrm{v}=0.209467, \mathrm{t}_{3}=0.800002, \mathrm{C}_{\mathrm{S}}=10.32, \mathrm{TC}_{1}=96.5648$.

Case 2: $\left(\mathrm{t}_{3}<\mathrm{M} \leq \mathrm{T}\right)$, Using mathematical software Mathematica 7 the output results are as follows: $\mathrm{T}=1.52193, \mathrm{v}=0.233164, \mathrm{t}_{3}=0.800001, \mathrm{C}_{\mathrm{S}}=10.30, \mathrm{TC}_{1}=90.8913$.

Case 3: $(M>T)$, Using mathematical software Mathematica 7 the output results are as follows: $\mathrm{T}=2.64105, \mathrm{v}=0.629393, \mathrm{t}_{3}=0.800475, \mathrm{C}_{\mathrm{S}}=10.13, \mathrm{TC}_{1}=50.9644$.

\section{Sensitivity Analysis}

Corresponding to different associated parameters, a sensitivity analysis is carried out to check the stability of the model. The analysis has been done with the parameters $M, \alpha, \beta, \mu, W$ taking one parameter at a time and other variables unchanged and is shown in Table 1, Table 2 and Table 3. 
Table 1

Case: $\mathrm{M} \leq \mathrm{t}_{3}<\mathrm{T}$

\begin{tabular}{|c|c|c|c|c|c|}
\hline$\%$ variation in $\mathrm{M}$ & $\mathrm{M}$ & $\mathrm{V}$ & $\mathrm{C}_{\mathrm{S}}$ & $\mathrm{T}$ & $\mathrm{TC}_{1}$ \\
\hline$-20 \%$ & 0.20 & 0.212076 & 10.39 & 1.57032 & 97.0438 \\
\hline$-15 \%$ & 0.2125 & 0.211429 & 10.37 & 1.56911 & 96.9242 \\
\hline$-10 \%$ & 0.225 & 0.210778 & 10.35 & 1.5679 & 96.8045 \\
\hline$-5 \%$ & 0.2375 & 0.210124 & 10.34 & 1.56668 & 96.6847 \\
\hline $0 \%$ & 0.25 & 0.21 & 10.32 & 1.565 & 96.5648 \\
\hline $5 \%$ & 0.2625 & 0.208806 & 10.31 & 1.56425 & 96.4448 \\
\hline $10 \%$ & 0.275 & 0.208141 & 10.30 & 1.56304 & 96.3247 \\
\hline $15 \%$ & 0.2875 & 0.207473 & 10.29 & 1.56182 & 96.2045 \\
\hline $20 \%$ & 0.3 & 0.206802 & 10.27 & 1.5606 & 96.0843 \\
\hline$\%$ variation in $\alpha$ & $\alpha$ & $\mathrm{V}$ & $\mathrm{C}_{\mathrm{S}}$ & $\mathrm{T}$ & $\mathrm{TC}_{1}$ \\
\hline$-20 \%$ & 0.048 & 0.209361 & 10.37 & 1.56799 & 96.813 \\
\hline$-15 \%$ & 0.051 & 0.209385 & 10.36 & 1.56741 & 96.7562 \\
\hline$-10 \%$ & 0.054 & 0.209411 & 10.34 & 1.5668 & 96.6958 \\
\hline$-5 \%$ & 0.057 & 0.209438 & 10.33 & 1.56615 & 96.632 \\
\hline $0 \%$ & 0.06 & 0.21 & 10.32 & 1.565 & 96.5648 \\
\hline $5 \%$ & 0.063 & 0.209497 & 10.30 & 1.56475 & 96.494 \\
\hline $10 \%$ & 0.066 & 0.209528 & 10.28 & 1.564 & 96.4198 \\
\hline $15 \%$ & 0.069 & 0.209561 & 10.27 & 1.56321 & 96.3421 \\
\hline $20 \%$ & 0.072 & 0.209595 & 10.26 & 1.56238 & 96.2608 \\
\hline$\%$ variation in $\beta$ & $\beta$ & $\mathrm{V}$ & $\mathrm{C}_{\mathrm{S}}$ & $\mathrm{T}$ & $\mathrm{TC}_{1}$ \\
\hline$-20 \%$ & 0.0080 & 0.209466 & 10.25 & 1056545 & 96.563 \\
\hline$-15 \%$ & 0.0085 & 0.209467 & 10.28 & 1.56546 & 96.5634 \\
\hline$-5 \%$ & 0.0095 & 0.209467 & 10.30 & 1056547 & 96.5643 \\
\hline $0 \%$ & 0.01 & 0.21 & 10.32 & 10565 & 96.5648 \\
\hline $5 \%$ & 0.0105 & 0.209467 & 10.34 & 1.56547 & 96.5652 \\
\hline $15 \%$ & 0.0115 & 0.209467 & 10.36 & 1.56548 & 96.5661 \\
\hline $20 \%$ & 0.012 & 0.209467 & 10.39 & 1.56549 & 96.5665 \\
\hline$\%$ variation in $\mu$ & $\mu$ & $\mathrm{V}$ & $\mathrm{C}_{\mathrm{S}}$ & $\mathrm{T}$ & $\mathrm{TC}_{1}$ \\
\hline$-20 \%$ & 16 & 0.288898 & 10.26 & 1.68971 & 87.4423 \\
\hline$-15 \%$ & 17 & 0.270418 & 10.28 & 1.65509 & 89.8131 \\
\hline$-5 \%$ & 19 & 0.230654 & 10.30 & 1.59324 & 94.3693 \\
\hline $0 \%$ & 20 & 0.21 & 10.32 & 1.565 & 96.5648 \\
\hline $5 \%$ & 21 & 0.18731 & 10.34 & 1.53951 & 98.7107 \\
\hline $15 \%$ & 23 & 0.224061 & 10.36 & 1.49219 & 102.876 \\
\hline $20 \%$ & 24 & 0.197361 & 10.39 & 1.47069 & 104.894 \\
\hline$\%$ variation in $\mathrm{W}$ & $\mathrm{W}$ & $\mathrm{V}$ & $\mathrm{C}_{\mathrm{S}}$ & $\mathrm{T}$ & $\mathrm{TC}_{1}$ \\
\hline$-20 \%$ & 325 & 0.464319 & 10.24 & 1.38236 & 79.4442 \\
\hline$-15 \%$ & 425 & 0.162829 & 10.28 & 1.49239 & 89.4711 \\
\hline$-5 \%$ & 475 & 0.175646 & 10.30 & 1.54182 & 94.236 \\
\hline $0 \%$ & 500 & 0.21 & 10.32 & 1.565 & 96.5648 \\
\hline $5 \%$ & 525 & 0.239235 & 10.40 & 1.58846 & 98.8582 \\
\hline $15 \%$ & 575 & 0.268156 & 10.45 & 1.63291 & 103.345 \\
\hline $20 \%$ & 600 & 0.210269 & 10.48 & 1.65537 & 105.537 \\
\hline
\end{tabular}


Table 2

Case: $\mathrm{t}_{3}<\mathrm{M}<\mathrm{T}$

\begin{tabular}{|c|c|c|c|c|c|}
\hline$\%$ variation in $\mathrm{M}$ & $\mathrm{M}$ & $\mathrm{V}$ & $\mathrm{C}_{\mathrm{S}}$ & $\mathrm{T}$ & $\mathrm{TC}_{2}$ \\
\hline$-20 \%$ & 0.20 & 0.232929 & 10.40 & 1.52145 & 90.9673 \\
\hline$-15 \%$ & 0.2125 & 0.232987 & 10.36 & 1.52157 & 90.9481 \\
\hline$-10 \%$ & 0.225 & 0.233045 & 10.34 & 1.52169 & 90.929 \\
\hline$-5 \%$ & 0.2375 & 0.233104 & 10.32 & 1.52181 & 90.9101 \\
\hline $0 \%$ & 0.25 & 0.233164 & 10.30 & 1.52193 & 90.8913 \\
\hline $5 \%$ & 0.2625 & 0.233225 & 10.28 & 1.52206 & 90.8727 \\
\hline $10 \%$ & 0.275 & 0.233287 & 10.26 & 1.52218 & 90.8543 \\
\hline $15 \%$ & 0.2875 & 0.233349 & 10.24 & 1.52231 & 90.836 \\
\hline $20 \%$ & 0.3 & 0.233412 & 10.20 & 1.52244 & 90.8179 \\
\hline$\%$ variation in $\alpha$ & $\alpha$ & $\mathrm{V}$ & $\mathrm{C}_{\mathrm{S}}$ & $\mathrm{T}$ & $\mathrm{TC}_{2}$ \\
\hline$-20 \%$ & 0.048 & 0.233004 & 10.34 & 1.52466 & 91.1466 \\
\hline$-15 \%$ & 0.051 & 0.233041 & 10.33 & 1.52403 & 91.0881 \\
\hline$-10 \%$ & 0.054 & 0.23308 & 10.32 & 1.52337 & 91.0261 \\
\hline$-5 \%$ & 0.057 & 0.233121 & 10.31 & 1.52267 & 90.9605 \\
\hline $0 \%$ & 0.06 & 0.233164 & 10.30 & 1.52193 & 90.8913 \\
\hline $5 \%$ & 0.063 & 0.233209 & 10.29 & 1.52115 & 90.8186 \\
\hline $10 \%$ & 0.066 & 0.233257 & 10.28 & 1.52034 & 90.7422 \\
\hline $15 \%$ & 0.069 & 0.233306 & 10.27 & 1.51948 & 90.6623 \\
\hline $20 \%$ & 0.072 & 0.233357 & 10.26 & 1.51859 & 90.5787 \\
\hline$\%$ variation in $\beta$ & $\beta$ & $\mathrm{V}$ & $\mathrm{C}_{\mathrm{S}}$ & $\mathrm{T}$ & $\mathrm{TC}_{2}$ \\
\hline$-20 \%$ & 0.0080 & 0.233164 & 10.31 & 1.52191 & 90.8895 \\
\hline$-15 \%$ & 0.0085 & 0.233164 & 10.32 & 1.52192 & 90.89 \\
\hline$-5 \%$ & 0.0095 & 0.233164 & 10.32 & 1.52193 & 90.8909 \\
\hline $0 \%$ & 0.01 & 0.233164 & 10.30 & 1.52193 & 90.8913 \\
\hline $5 \%$ & 0.0105 & 0.233164 & 10.33 & 1.52194 & 90.8918 \\
\hline $15 \%$ & 0.0115 & 0.233164 & 10.34 & 1.52195 & 90.8927 \\
\hline $20 \%$ & 0.012 & 0.233164 & 10.35 & 1.52195 & 90.8931 \\
\hline$\%$ variation in $\mu$ & $\mu$ & $\mathrm{V}$ & $\mathrm{C}_{\mathrm{S}}$ & $\mathrm{T}$ & $\mathrm{TC}_{2}$ \\
\hline$-20 \%$ & 16 & 0.243621 & 10.27 & 1.64455 & 82.1459 \\
\hline$-15 \%$ & 17 & 0.281719 & 10.29 & 1.60968 & 84.4194 \\
\hline$-5 \%$ & 19 & 0.254041 & 10.30 & 1.54904 & 88.7858 \\
\hline $0 \%$ & 20 & 0.233164 & 10.30 & 1.52193 & 90.8913 \\
\hline $5 \%$ & 21 & 0.210584 & 10.36 & 1.49662 & 92.9506 \\
\hline $15 \%$ & 23 & 0.161105 & 10.38 & 1.45061 & 96.9425 \\
\hline $20 \%$ & 24 & 0.133974 & 10.40 & 1.42963 & 98.8804 \\
\hline$\%$ variation in $\mathrm{W}$ & $\mathrm{W}$ & $\mathrm{V}$ & $\mathrm{C}_{\mathrm{S}}$ & $\mathrm{T}$ & $\mathrm{TC}_{2}$ \\
\hline$-20 \%$ & 325 & 0.265854 & 10.26 & 1.34846 & 75.2939 \\
\hline$-15 \%$ & 425 & 0.105896 & 10.28 & 1.45194 & 84.4201 \\
\hline$-5 \%$ & 475 & 0.196621 & 10.30 & 1.49925 & 88.7693 \\
\hline $0 \%$ & 500 & 0.233164 & 10.30 & 1.52193 & 90.8913 \\
\hline $5 \%$ & 525 & 0.255453 & 10.35 & 1.54409 & 92.9808 \\
\hline $15 \%$ & 575 & 0.237146 & 10.38 & 1.58701 & 97.0676 \\
\hline $20 \%$ & 600 & 0.237146 & 10.40 & 1.60777 & 99.0641 \\
\hline
\end{tabular}


Table 3

Case: $\mathrm{M}>\mathrm{T}$

\begin{tabular}{|c|c|c|c|c|c|}
\hline$\%$ variation in $\mathrm{M}$ & $\mathrm{M}$ & $\mathrm{V}$ & $\mathrm{C}_{\mathrm{S}}$ & $\mathrm{T}$ & $\mathrm{TC}_{3}$ \\
\hline$-20 \%$ & 0.20 & 0.631124 & 10.01 & 2.68024 & 48.7894 \\
\hline$-15 \%$ & 0.2125 & 0.630693 & 10.08 & 2.67038 & 49.3402 \\
\hline$-10 \%$ & 0.225 & 0.630261 & 10.10 & 2.66056 & 49.8863 \\
\hline$-5 \%$ & 0.2375 & 0.629828 & 10.12 & 2.65078 & 50.4276 \\
\hline $0 \%$ & 0.25 & 0.629393 & 10.13 & 2.64105 & 50.9644 \\
\hline $5 \%$ & 0.2625 & 0.628956 & 10.14 & 2.63137 & 51.4965 \\
\hline $10 \%$ & 0.275 & 0.628578 & 10.15 & 2.62173 & 52.0241 \\
\hline $15 \%$ & 0.2875 & 0.628079 & 10.16 & 2.61214 & 52.5473 \\
\hline $20 \%$ & 0.3 & 0.627638 & 10.17 & 2.6026 & 53.066 \\
\hline$\%$ variation in $\alpha$ & $\alpha$ & $\mathrm{V}$ & $\mathrm{C}_{\mathrm{S}}$ & $\mathrm{T}$ & $\mathrm{TC}_{3}$ \\
\hline$-20 \%$ & 0.048 & 0.629083 & 10.22 & 2.64152 & 51.1025 \\
\hline$-15 \%$ & 0.051 & 0.629155 & 10.20 & 2.6414 & 51.0712 \\
\hline$-10 \%$ & 0.054 & 0.629231 & 10.17 & 2.64128 & 51.0378 \\
\hline$-5 \%$ & 0.057 & 0.62931 & 10.15 & 2.64116 & 51.0022 \\
\hline $0 \%$ & 0.06 & 0.629393 & 10.13 & 2.64105 & 50.9644 \\
\hline $5 \%$ & 0.063 & 0.629478 & 10.11 & 2.64095 & 50.9243 \\
\hline $10 \%$ & 0.066 & 0.629566 & 10.09 & 2.64087 & 50.882 \\
\hline $15 \%$ & 0.069 & 0.629657 & 10.07 & 2.6408 & 50.8372 \\
\hline $20 \%$ & 0.072 & 0.62975 & 10.05 & 2.64075 & 50.79 \\
\hline$\%$ variation in $\beta$ & $\beta$ & $\mathrm{V}$ & $\mathrm{C}_{\mathrm{S}}$ & $\mathrm{T}$ & $\mathrm{TC}_{3}$ \\
\hline$-20 \%$ & 0.0080 & 0.629395 & 10.22 & 2.64105 & 50.9634 \\
\hline$-15 \%$ & 0.0085 & 0.629328 & 10.20 & 2.64114 & 50.9994 \\
\hline$-5 \%$ & 0.0095 & 0.62932 & 10.18 & 2.64115 & 51.0037 \\
\hline $0 \%$ & 0.01 & 0.629393 & 10.13 & 2.64105 & 50.9644 \\
\hline $5 \%$ & 0.0105 & 0.629392 & 10.15 & 2.64105 & 50.9646 \\
\hline $15 \%$ & 0.0115 & 0.629391 & 10.17 & 2.64105 & 50.9651 \\
\hline $20 \%$ & 0.012 & 0.629391 & 10.19 & 2.64105 & 50.9654 \\
\hline$\%$ variation in $\mu$ & $\mu$ & $\mathrm{V}$ & $\mathrm{C}_{\mathrm{S}}$ & $\mathrm{T}$ & $\mathrm{TC}_{3}$ \\
\hline$-20 \%$ & 16 & 0.617807 & 10.20 & 2.2989 & 60.0347 \\
\hline$-15 \%$ & 17 & 0.604202 & 10.18 & 2.41641 & 58.412 \\
\hline$-5 \%$ & 19 & 0.627078 & 10.16 & 2.50576 & 55.1593 \\
\hline $0 \%$ & 20 & 0.629393 & 10.13 & 2.64105 & 50.9644 \\
\hline $5 \%$ & 21 & 0.807184 & 10.14 & 1.66519 & 70.7492 \\
\hline $15 \%$ & 23 & 0.811206 & 10.15 & 1.70868 & 69.4602 \\
\hline $20 \%$ & 24 & 0.814062 & 10.16 & 1.73548 & 68.3626 \\
\hline$\%$ variation in $\mathrm{W}$ & $\mathrm{W}$ & $\mathrm{V}$ & $\mathrm{C}_{\mathrm{S}}$ & $\mathrm{T}$ & $\mathrm{TC}_{3}$ \\
\hline$-20 \%$ & 325 & 0.790605 & 10.21 & 1.67133 & 43.0109 \\
\hline$-15 \%$ & 425 & 0.678981 & 10.18 & 2.78981 & 44.4998 \\
\hline$-5 \%$ & 475 & 0.621668 & 10.15 & 2.6738 & 49.1142 \\
\hline $0 \%$ & 500 & 0.629393 & 10.13 & 2.64105 & 50.9644 \\
\hline $5 \%$ & 525 & 0.619647 & 10.10 & 2.76927 & 50.526 \\
\hline $15 \%$ & 575 & 0.671908 & 10.09 & 2.62214 & 50.0103 \\
\hline $20 \%$ & 600 & 0.591017 & 10.01 & 2.74682 & 50 \\
\hline
\end{tabular}




\subsection{Observations}

\section{Case 1: $\left(M \leq t_{3}<T\right)$}

1. An increase in $M$ and $\alpha$ reduces $v, T, C_{S}$ and $T C_{1}$.

2. An increase in $\beta$ results in decrement of $v$ but increases in $C_{S}, T$ and $\mathrm{TC}_{1}$.

3. An increase in $\mu$ results in decrement of $v, T$ but increment in $C_{S}$ and $T C_{l}$.

4. An increase in $W$ result in increment in $v, C_{S}, T$ and $T C_{l}$.

Case 2: $\left(\mathrm{t}_{3}<M \leq T\right)$

1. An increase in $\beta$ yields an increment in $T, T C_{2}$ and $C_{S}$ but $v$ remains unchanged.

2. An increase in $\mu$ results in decrement of $v, T$ but increment in $C_{S}$ and $T C_{2}$.

3. An increase in $W$ results in increment in $v, C_{S}, T$ and $T C_{2}$.

4. An increase in $\alpha$ results in increment in $v$ but decrement in $C_{S}, T$ and $T C_{2}$.

5. An increase in $M$ result in increment of $v$ and $T$ but decrement in $C_{S}$ and $T C_{2}$.

\section{Case 3: $(M>T)$}

1. An increase in $M$ results in increment of $C_{S}$ and $T C_{3}$ but reduces $v$ and $T$.

2. An increase in $\alpha$ results in increment in $v$ but reduces $\mathrm{C}_{\mathrm{S}}, \mathrm{T}$ and $\mathrm{TC}_{3}$.

3. An increase in $\beta$ results in decrement of $v, C_{S}$ but increment in $T C_{3}$ and $T$ remains unchanged.

4. An increase in $\mu$ results in decrement of $T$ but increment in $v, C_{S}$ and $T C_{3}$.

5. An increase in $W$ results in decrement in $v, C_{S}$ and $T C_{3}$ and increment in $T$.

\section{Conclusion}

In this paper we have presented an integrated production inventory model under reliability consideration. It is impossible to consider that every production system is perfect. The demand rate was taken as increasing function of time which shows a very realistic phenomenon. The effect of permissible delay in payment and inflation has also been considered. A numerical example has been shown to illustrate the model. The model is optimized and the convexity of the model is shown. A sensitivity analysis is also performed to check the stability of the model. For future scope the model can be extended for stochastic demand rate and with learning and forgetting effects for production and manufacturing.

\section{Acknowledgement}

The authors would like to thank the anonymous referees for constructive comments on earlier version of this paper. 


\section{References}

Buzacott, J. A. (1975). Economic order quantities with inflation. Operational research quarterly, 553-558.

Cheng, T. C. E. (1989). An economic order quantity model with demand-dependent unit cost. European Journal of Operational Research, 40(2), 252-256.

Dey, J. K., Mondal, S. K., \& Maiti, M. (2008). Two storage inventory problem with dynamic demand and interval valued lead-time over finite time horizon under inflation and time-value of money. European Journal of Operational Research, 185(1), 170-194.

Lin, L. C., \& Hou, K. L. (2005). An inventory system with investment to reduce yield variability and set-up cost. Journal of the Operational Research Society, 56(1), 67-74.

Leung, K. N. F. (2007). A generalized geometric-programming solution to "An economic production quantity model with flexibility and reliability considerations". European Journal of Operational Research, 176(1), 240-251.

Liang, Y., \& Zhou, F. (2011). A two-warehouse inventory model for deteriorating items under conditionally permissible delay in payment. Applied Mathematical Modelling, 35(5), 22212231.

Hadidi, L. A., Al-Turki, U. M., \& Rahim, M. A. (2011). An integrated cost model for production scheduling and perfect maintenance. International Journal of Mathematics in Operational Research, 3(4), 395-413.

Maity, K., \& Maiti, M. (2009). Optimal inventory policies for deteriorating complementary and substitute items. International Journal of Systems Science, 40(3), 267-276.

Pal, P., Bhunia, A. K., \& Goyal, S. K. (2007). On optimal partially integrated production and marketing policy with variable demand under flexibility and reliability considerations via Genetic Algorithm. Applied mathematics and computation, 188(1), 525-537.

Panda, D., \& Maiti, M. (2009). Multi-item inventory models with price dependent demand under flexibility and reliability consideration and imprecise space constraint: A geometric programming approach. Mathematical and Computer Modelling, 49(9), 1733-1749.

Park, K. S. (1983). An integrated production-inventory model for decaying raw materials. International Journal of Systems Science, 14(7), 801-806.

Shah, N. H. (2006). Inventory model for deteriorating items and time value of money for a finite time horizon under the permissible delay in payments. International Journal of Systems Science, 37(1), 9-15.

Singh, S. R. (2009). Integrated Vendor-Buyer Cooperative Model with Multivariate Demand and Progressive Credit Period. IUP Journal of Operations Management, 8(2), 36-50.

Singh, N., Vaish, B., \& Singh, S. R. (2010). An EOQ model with Pareto distribution for deterioration, Trapezoidal type demand and backlogging under trade credit policy. The IUP Journal of Computational Mathematics, 3(4), 30-53.

Singh, S. R., Gupta, V., \& Bansal, P. (2013). EOQ model with volume agility, variable demand rate, Weibull deterioration rate and inflation. International Journal of Computer Applications, 72(23), 1-6.

Singh, S., \& Prasher, L. (2014). A production inventory model with flexible manufacturing, random machine breakdown and stochastic repair time. International Journal of Industrial Engineering Computations, 5(4), 575-588.

Singh, S. R., \& Saxena, P. (2014, November). Fuzzy warehouse inventory model for items with imperfect quality under trade credit policy and inflationary conditions. In Computational Intelligence on Power, Energy and Controls with their impact on Humanity (CIPECH), 2014 Innovative Applications of (pp. 69-74). IEEE.

Singhal, S, Singh, S. (2013). Volume flexible multi items inventory system with imprecise environment. International Journal of Industrial Engineering Computations, 4(4), 457-468.

Soni, H., Gor, A. S., \& Shah, N. H. (2006). An EOQ model for progressive payment scheme under DCF approach. Asia-Pacific Journal of Operational Research, 23(04), 509-524. 
Soni, H. N., \& Patel, K. A. (2014). Optimal policies for vendor-buyer inventory system involving defective items with variable lead time and service level constraint. International Journal of Mathematics in Operational Research, 6(3), 316-343.

Tayal, S., Singh, S. R., Chauhan, A., \& Sharma, R. (2014). A Deteriorating Production inventory Problem with Space Restriction. Journal of Information and Optimization Sciences, 35(3), 203-229.

Yadav, D., Pundir, S., \& Kumari, R. (2010). A fuzzy multi-item production model with reliability and flexibility under limited storage capacity with deterioration via geometric programming. International Journal of Mathematics in Operational Research, 3(1), 78-98.

Yan, H., \& Cheng, T. C. E. (1998). Optimal production stopping and restarting times for an EOQ model with deteriorating items. Journal of the Operational Research Society, 49(12), 12881295.

Yang, H. L. (2006). Two-warehouse partial backlogging inventory models for deteriorating items under inflation. International Journal of Production Economics, 103(1), 362-370. 